\title{
Peculiarities of Cirrhosis due to Nonalcoholic Steatohepatitis (NASH)
}

\author{
Omar El-Sherif, $\mathrm{PhD}^{1} \quad$ M.J. Armstrong, $\mathrm{PhD}^{1,2}$ \\ ${ }^{1}$ Liver Unit, Queen Elizabeth University Hospital Birmingham, \\ Birmingham, United Kingdom \\ ${ }^{2}$ NIHR Birmingham Biomedical Research Centre, Centre for Liver \\ Address for correspondence Omar El-Sherif, PhD, Liver Unit, 3rd floor, \\ Nuffield House, Queen Elizabeth University Hospital Birmingham, \\ Birmingham, United Kingdom (e-mail: elsherio@tcd.ie).
} Research, University of Birmingham, Birmingham, United Kingdom

Semin Liver Dis 2020;40:1-10.

\begin{abstract}
Keywords

- nonalcoholic fatty liver

- steatohepatitis

- cirrhosis

- challenges

The prevalence of cirrhosis due to nonalcoholic steatohepatitis (NASH) has increased 2.5-fold in the United States in the last decade. These patients pose new challenges to hepatologists given their older age and higher frequency of coexisting metabolic diseases such as obesity and diabetes compared with other etiologies of liver disease. Patients with NASH cirrhosis are at higher risk for renal and cardiovascular disease, and the presence of these extrahepatic comorbidities has a significant impact on outcomes and survival. This review outlines how NASH cirrhosis differs from other etiologies of cirrhosis including natural history, noninvasive assessment, and the challenges in the management of the complications of cirrhosis including hepatic encephalopathy and hepatocellular carcinoma. Nutritional assessment and the impact of sarcopenic obesity and frailty in this population, and strategies to address the latter, are discussed. This review also addresses liver transplantation in patients with NASH cirrhosis in relation to assessment and posttransplant care.
\end{abstract}

Nonalcoholic fatty liver disease (NAFLD) encompasses a spectrum of manifestations ranging from simple nonalcoholic fatty liver to nonalcoholic steatohepatitis (NASH) with progressive fibrosis and ultimately cirrhosis in a small proportion of patients. Data from the National Health and Nutrition Examination Survey suggest that there has been a 2- and 2.5-fold increase in the overall prevalence of NAFLD-associated advanced fibrosis and cirrhosis in the last decade alone in the United States, respectively. ${ }^{1}$ The frequency of liver transplantation for NASH cirrhosis has also increased 10-fold in the same time period. ${ }^{2,3}$ These patients, however, pose new challenges in the field of hepatology when compared with other, more established liver diseases. These challenges include the multimodal pathogenesis, variable natural history, diagnostic tools, lack of pharmaceutical therapies, and the high frequency of coexistent metabolic diseases (i.e., diabetes, obesity). The latter, when severe (i.e., diabetic retinopathy/nephropathy; body mass index $[\mathrm{BMI}]>40 \mathrm{~kg} / \mathrm{m}^{2}$ ), not only limit the accuracy of radiological assessment (i.e., transient elastography [TE], ultra- sound cancer surveillance), but also question the patients' fitness for surgical procedures and liver transplantation.

This review summarizes specific aspects of cirrhosis due to NASH and how it differs from other etiologies of cirrhosis including natural history, noninvasive assessment, and the challenges in the management of the complications of cirrhosis, extrahepatic comorbidities, and liver transplantation (-Fig. 1).

\section{Natural History of Cirrhosis Due to NASH}

Long-term prospective natural history data in patients with NASH cirrhosis are limited. There are several reasons to account for this. First, NASH as a disease entity has only gained worldwide recognition in the last couple of decades, despite the initial reports being as early as 1980 by Ludwig and his American colleagues. ${ }^{4}$ Second, many patients with NASH cirrhosis were historically diagnosed as having cryptogenic ("unknown cause") cirrhosis, which subsequently questions the validity of grouping these individuals together 


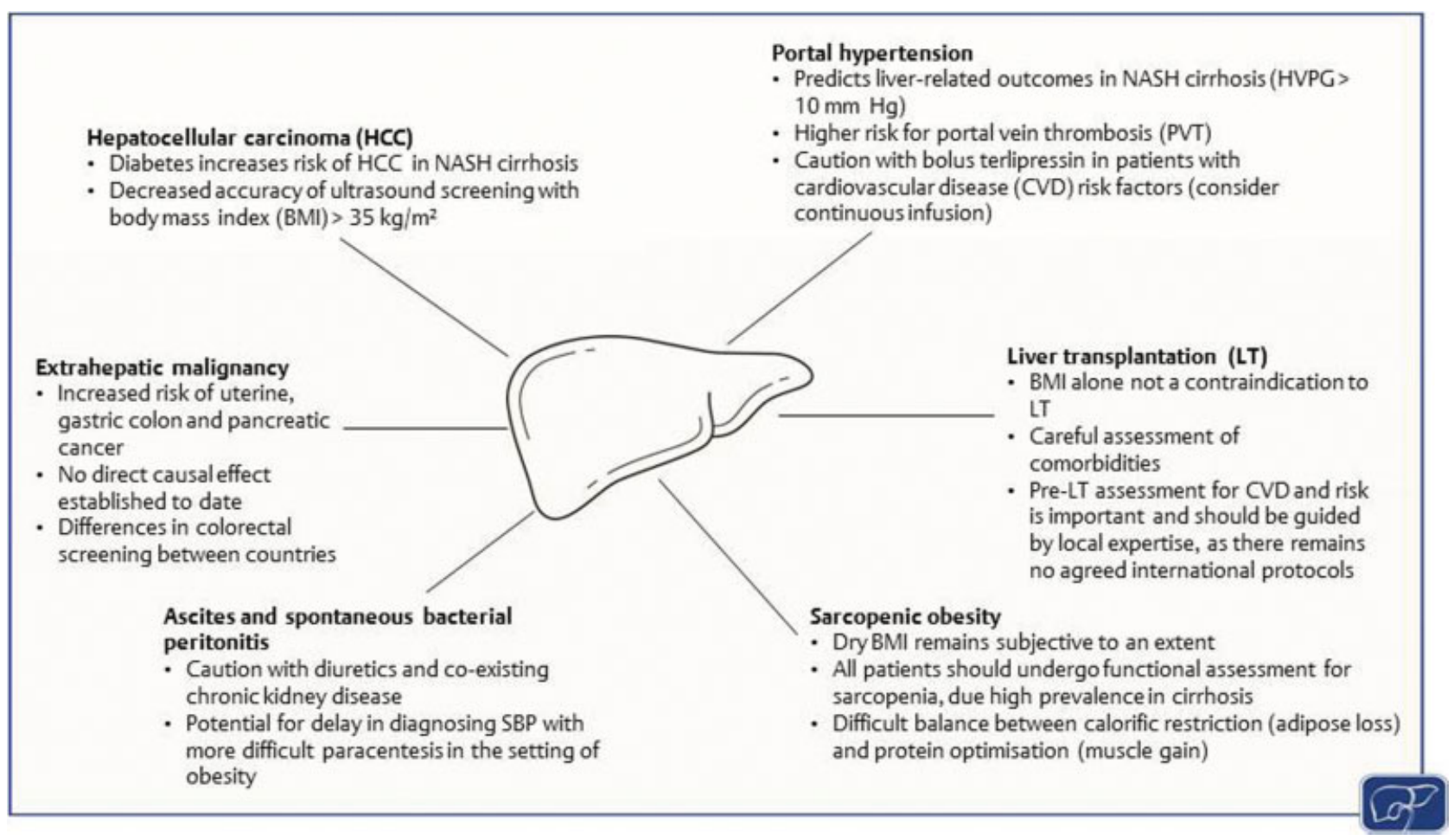

Fig. 1 Challenges with cirrhosis secondary to nonalcoholic steatohepatitis (NASH).

for retrospective cohort studies. Third, NASH in the majority of cases is a slowly progressive disease compared with many other etiologies of liver disease, in that it takes at least 7 years on average for fibrosis to progress by one stage in $\mathrm{NASH}^{5}$ Longer follow-up studies are still required to accurately capture the development of cirrhosis-related complications in patients with NASH. - Table 1 summarizes follow-up studies (five retrospective $[n=18-74]$; one randomized controlled trial $[n=256]$ ) that included patients with compensated NASH cirrhosis at baseline. One of the largest studies to date is by Hagström et al, consisting of 646 biopsy-proven patients with NAFLD and 20 years' followup. They consolidated the fact that fibrosis (rather than
NASH) is the best predictor of liver-related mortality and that of those with cirrhosis $(n=20)$, almost $50 \%$ decompensated in the study timeframe. ${ }^{6}$ However, these data sets remain limited by the small numbers of patients with cirrhosis, the selection bias of cases in tertiary care centers, the lack of individual patient data during follow-up, as well as the retrospective design. Bosch et al's analysis of the phase $2 \mathrm{~b}$ trial of simtuzumab (monoclonal antibody against lysyl oxidase-like 2) highlights the potential for future NASH cirrhosis trials (especially placebo arms or noneffective intervention arms) to aid with our understanding of disease progression and predictors of decompensation (i.e., hepatic venous pressure gradient [HVPG]). ${ }^{7}$ It is, however, important

Table 1 Outcome studies in patients with compensated NASH cirrhosis

\begin{tabular}{|c|c|c|c|c|c|}
\hline Study & Follow-up & $n$ & Study design & $\begin{array}{l}\text { Diagnosis of } \\
\text { cirrhosis }\end{array}$ & Outcomes \\
\hline Hagström et al ${ }^{6}$ & $\begin{array}{l}19.9 \text { y } \\
\text { (mean) }\end{array}$ & 20 & Retrospective & Biopsy & $\begin{array}{l}\text { Liver decompensation in } 45 \% \text {; } 10 \% \text { developed } \\
\text { decompensation within } 5.6 \mathrm{y}\end{array}$ \\
\hline Sanyal et al ${ }^{9}$ & $\sim 12 \mathrm{y}$ & 74 & Retrospective & Biopsy & 10 -y mortality rate $4 \%$ \\
\hline Angulo et al ${ }^{84}$ & $\begin{array}{l}12.6 \mathrm{y} \\
\text { (median) }\end{array}$ & 18 & Retrospective & Biopsy & $\begin{array}{l}\text { Liver decompensation or transplantation in } \\
77.8 \% \text { (transplant-free survival 22.2\%) }\end{array}$ \\
\hline Younossi et al ${ }^{85}$ & $\begin{array}{l}12.2 \mathrm{y} \\
\text { (median) }\end{array}$ & 22 & Retrospective & Biopsy & $\begin{array}{l}\text { Liver-related mortality in } 41 \% \text {; all-cause } \\
\text { mortality } 59 \%\end{array}$ \\
\hline Sebastiani et al ${ }^{86}$ & $\begin{array}{l}5 \text { y } \\
\text { (median) }\end{array}$ & 22 & Retrospective & Biopsy & Liver-related mortality in $13.6 \%$ \\
\hline Bosch et al ${ }^{7 a}$ & $\begin{array}{l}2.2 \mathrm{y} \\
\text { (median) }\end{array}$ & 256 & $\begin{array}{l}\text { Prospective Phase } \\
\text { 2b clinical trial }\end{array}$ & Biopsy & $\begin{array}{l}\text { Liver-related events in } 19 \% ; 1 \% \text { mortality in } \\
\text { the follow-up period }\end{array}$ \\
\hline
\end{tabular}

Abbreviations: NASH, nonalcoholic steatohepatitis; y, years.

a Unpublished data presented in abstract format. 
to acknowledge that the patient population was selected for trial purposes only (i.e., bias), follow-up was only reported at 2.2 years, and the data are published in abstract format only at present.

Patients with NASH cirrhosis are older than patients with cirrhosis of other etiologies, ${ }^{8}$ which is not surprising given the risk factors for NAFLD (components of metabolic syndrome) tend to increase in prevalence with advancing age. Not only are older patients with cirrhosis more susceptible to cancer and death, but their management poses additional challenges with regards to suitability for transplantation, tolerance of polypharmacy (i.e., diuretics, antihypertensives), adoption of lifestyle changes (i.e., exercise), and multiple comorbidities. ${ }^{8}$ The risk of decompensation, however, appears to be lower in patients with NASH cirrhosis compared with non-NASH cirrhosis (mainly viral, alcohol) patients. Patients with NASH cirrhosis have increased cardiovascular morbidity and mortality, but lower liver-related mortality in the compensated state (Child-Pugh A) when compared with patients with hepatitis $C$ virus (HCV)-related cirrhosis. ${ }^{9}$ In fact, cardiovascular disease is the most common cause of death in patients with compensated cirrhosis due to $\mathrm{NASH}$. It is only in patients with decompensated NASH cirrhosis where liver disease overtakes cardiovascular disease as the leading cause of mortality. ${ }^{10}$

In the only published prospective data set (abstract only) of patients with compensated NASH cirrhosis, $19 \%$ of patients experienced liver-related clinical events (ascites, encephalopathy, variceal hemorrhage) during a follow-up period of 27 months. $^{7}$ The biggest predictor of decompensation and liver-related events was baseline HVPG, with low rates of decompensation in patients without clinically significant portal hypertension at baseline (HVPG $<10 \mathrm{~mm} \mathrm{Hg}){ }^{7}$ Once decompensation (Child-Pugh $\mathrm{B} / \mathrm{C}$ ) has developed, overall survival and liver-related mortality appears to be the same in NASH cirrhosis as in other cirrhosis etiologies. ${ }^{11}$

There are no longitudinal studies examining the effect of ongoing alcohol consumption on disease severity or the natural history of NASH, especially in the presence of cirrhosis. There are conflicting data from cross-sectional studies on the effect of mild to moderate alcohol intake on fibrosis progression in patients with NASH without cirrhosis. ${ }^{12,13}$ However, patients with NASH cirrhosis as with all other cirrhosis etiologies should be advised to completely abstain from alcohol consumption.

\section{Pharmacotherapy and Clinical Trials for NASH Cirrhosis}

At present, there are no therapies approved by the European Medicines Agency or the Federal Drug Administration in the United States for the treatment of compensated NASH cirrhosis, although several agents are in phase 2 and 3 development. The choice of endpoints for these clinical trials has been the subject of much debate. ${ }^{14}$ Clinical trials with hard endpoints of decompensation, liver transplantation, or other liver-related events (i.e., variceal hemorrhage, hepatocellular carcinoma [HCC]) require follow-up periods of many years; especially in the absence of significant portal hypertension at baseline (exclusion criteria in most trials). As a result, regulatory approval frameworks have adopted surrogate endpoints (liver fibrosis stage on biopsy, magnetic resonance elastography) that may be achieved within a reasonably short time frame (i.e., 2 years).

The Stellar-4 phase 3 trial of selonsertib (an oral inhibitor of apoptosis signal-regulating kinase 1) in compensated NASH cirrhosis (biopsy confirmed) utilized a prespecified 48-week primary endpoint of $a \geq 1$-stage histologic improvement in kleiner fibrosis without worsening of NASH. There was no difference between selonsertib and placebo in this trial. ${ }^{15}$ As previously discussed, portal hypertension is an important predictor of liver-related outcomes in NASH cirrhosis. Emricasan, an oral pan-caspase inhibitor, failed to meet its primary (24-week endpoint) of reduction in HVPG in patients with NASH cirrhosis and a baseline $\mathrm{HVPG} \geq 12 \mathrm{~mm} \mathrm{Hg}$. ${ }^{16}$ It remains to be seen as to whether the treatment duration was too short to see an effect, as improvements in HVPG were only seen in studies with direct acting antiviral therapy in viral hepatitis $C$ after 12 to 24 months of viral suppression. ${ }^{17}$ However, the phase $2 \mathrm{~b}$ study of simtuzumab did not show any significant effect of simtuzumab on HVPG in patients with compensated cirrhosis after a longer period 96 weeks' follow-up. ${ }^{18}$ Other therapeutic trials targeting NASH cirrhosis are still ongoing with drug groups including glucagon-like peptide 1 (GLP-1) analogs and synthetic bile acid analogues (farnesoid-X receptor agonists).

\section{Noninvasive Assessment of Cirrhosis and Varices}

Transient elastography (Fibroscan; Echosens) for the noninvasive assessment of liver fibrosis has drastically reduced the need for liver biopsy. ${ }^{19}$ However, morbid obesity $\left(\mathrm{BMI}>35 \mathrm{~kg} / \mathrm{m}^{2}\right)$ and particularly increased waist circumference are limitations in obtaining reliable measurements with TE. ${ }^{20}$ The introduction of Fibroscan XL probe in 2009 overcame some of these limitations with a lower failure rate in patients with $\mathrm{BMI} \geq 30 \mathrm{~kg} / \mathrm{m}^{2}$ when compared with the conventional medium (M) probe. ${ }^{21} \mathrm{TE}$ is an accurate "rule-out" tool (good negative predictive value $>90 \%$ ) for cirrhosis in patients with $\mathrm{NASH}$, alcohol-related, and viral $\mathrm{B}$ and $\mathrm{C}$ hepatitis. However, unlike viral hepatitis, ${ }^{22}$ TE has a poor positive predictive value $(<40 \%)$ for the diagnosis of cirrhosis in patients with NASH. ${ }^{23}$ As a result, liver biopsy is still widely required to establish the diagnosis of cirrhosis in patients with NAFLD and liver stiffness $\geq 8 \mathrm{kPa}$ in the absence of other clinical or radiological signs of cirrhosis/portal hypertension. ${ }^{24}$ Furthermore, TE is not widely used in the United states and its practice is largely confined to European and Asian countries.

A recent advance is that TE can also be used in the risk stratification of clinically significant portal hypertension (HVPG $\geq 10 \mathrm{~mm} \mathrm{Hg}$ ) and thereby guide the need for endoscopy for variceal assessment in patients with cirrhosis. The Baveno VI guidelines proposed that patients with compensated cirrhosis with liver stiffness $<20 \mathrm{kPa}$ and a platelet count $>150,000 / \mu \mathrm{L}$ can avoid baseline screening endoscopy. ${ }^{25}$ The NAFLD cirrhosis 
criteria uses different cut-offs for the M probe (liver stiffness measurement $[\mathrm{LSM}]<30 \mathrm{kPa}$ and platelet count $>110,000 / \mu \mathrm{L})$ and XL probe (LSM $<25 \mathrm{kPa}$ and platelet $>110,000 / \mu \mathrm{L})$ to select patients that do not require screening endoscopy. ${ }^{26}$ While disease-specific criteria improve the negative predictive value for varices, only a small additional proportion of patients might be spared endoscopy with their use. ${ }^{27}$ Whether this small additional number of avoided endoscopies is enough to outweigh the simplicity of a single threshold of all patients with cirrhosis remains a matter of debate.

Prospective studies have also examined the role of spleen stiffness using elastography for the diagnosis of cirrhosis, but none of these studies have been specific to patients with NASH cirrhosis. ${ }^{28}$ Recent prospective data have also highlighted the utility of liver-to-spleen stiffness measurements using TE $(n=274)$ for variceal screening in compensated cirrhosis compared with conventional endoscopy $(n=274) .{ }^{29}$ In doing so, the group from Hong Kong highlighted that the number of screening endoscopies could be reduced by $50 \%$ using liver-tospleen stiffness measurements. However, as $85 \%$ of the cohort had viral hepatitis this would require further validation in patients with NASH cirrhosis.

\section{Nutrition and Exercise}

The importance of nutrition and exercise in liver disease and cirrhosis is increasingly recognized. Protein energy malnutrition, obesity, and in particular sarcopenic obesity impact on prognosis in cirrhosis and their presence is associated with reduced survival in patients with decompensated disease. ${ }^{30,31}$

Sarcopenia and obesity have previously been viewed as separate entities on opposite ends of the spectrum. However, sarcopenic obesity, defined as the combination of loss of skeletal muscle mass/function and gain in adipose tissue, is observed with increasing frequency in patients with cirrhosis. ${ }^{32}$ Patients with NASH cirrhosis are at high risk for sarcopenic obesity, by the nature of the coexistent metabolic syndrome. It presents a challenge for the patient in ensuring adequate nutrition to replenish muscle mass, without increasing excess adipose tissue stores. Paradoxically, patients with compensated NASH cirrhosis are frequently advised to lose weight to reduce the risk of disease progression, but in doing so can exacerbate sarcopenia if undertaken without a corresponding calculated increase in protein intake. Irrespective of disease etiology, obesity is an independent risk factor for disease progression and decompensation in patients with cirrhosis. ${ }^{33}$ In addition, patients with obesity and cirrhosis are at high risk for depletion of various fat-soluble, water-soluble vitamins and trace elements and should be supplemented appropriately.

Waist circumference (and waist-to-hip ratio), as measures of abdominal obesity, have repeatedly been shown to be a better predictor of NAFLD and severe liver disease than BMI. There remains, however, a paucity of data of the accuracy of waist circumference in predicting clinical outcomes in patients with compensated NASH cirrhosis. Accurately defining waist circumference and BMI remains a challenge in more advanced cirrhosis due to ascites, peripheral edema, and hepatic hydrothoraces. International guidelines recommend calculating dry
BMI by means of postparacentesis weight (which does not rule out peripheral edema) or the more subjective calculation of subtracting a percentage of weight based upon the severity of ascites (mild 5\%; moderate $10 \%$; severe $15 \%$ ), with an additional $5 \%$ subtracted if bilateral pedal edema is present. ${ }^{34}$ This is still not validated and the accuracy of BMI is repeatedly questioned when analyzing outcomes pre- and post-liver transplantation. ${ }^{35}$ Furthermore, the presence of sarcopenia in the setting of NASH cirrhosis, obesity, and/or fluid retention is often overlooked and therefore routinely all patients with NASH cirrhosis should undergo a functional assessment for sarcopenia. Hand grip strength (HGS) is an easy to perform, replicable, and reproducible screening test for muscle function and correlates with clinical outcome. HGS, together with measures of balance and chair stands, contribute to the overall liver frailty index. ${ }^{30}$ Even though this relatively new index of physical frailty correlates with hospital admissions, length of hospital stay, and mortality, it has not been validated outside of the United States or in specific diseases like NASH cirrhosis. Physical activity levels measured by average daily step counts are lower in patients with cirrhosis than in healthy controls, and low levels of physical activity in this group are associated with increased insulin resistance. ${ }^{36}$ This is particularly important in patients with NASH cirrhosis, who often have accompanying type 2 diabetes (T2D) and sedentary lifestyles, which have been the driving factors in the development of their disease for many years.

Patients with NASH cirrhosis require early involvement with clinical nutritionists with appropriate dietary expertise in managing patients with chronic liver disease. Weight loss of greater than $5 \%$, using a combination of hypocaloric diet and exercise has been shown to be an effective and safe treatment for NAFLD, ${ }^{37}$ with likely paralleled reduction in cardiovascular risk as seen in overweight and obese patients with T2D. ${ }^{38}$ The additional benefits of hypocaloric diet (500-1,000 calories/day) and exercise on reducing portal hypertension, as measured intricately by HVPG, has been recently reported in 50 obese patients (predominantly Child-Pugh A; 24\% NASH cirrhosis). Even though the intervention was only 16 weeks, the weight loss was maintained for 6 months with no detrimental effects on the liver disease severity (i.e., no decompensations, Model for End-Stage Liver Disease [MELD] stable). ${ }^{39}$ It remains to be seen whether exercise alone, in the absence of restrictive diets and weight loss, directly improves portal hypertension. Whether a hypocaloric diet and exercise is safe or efficacious in patients with decompensated NASH cirrhosis remains untested, but due to the higher rates of protein catabolism and poor glycogen storage, such calorific restriction in the community is likely to be harmful. In addition, the lifestyle changes required are not easy to implement without regular motivation and guidance from a health care professional. Patients with NASH cirrhosis are often older, and frequently have other comorbidities such as osteoarthritis and obesity that further limits their physical activity and ability to engage in aerobic cardiopulmonary exercises such as jogging and cycling. Advice alone to increase physical activity levels without an easy-to-follow plan with regular psychological re-enforcement is unlikely to be of benefit. 
There remains a lack of robust data available with regards to the best types of physical exercise (aerobic vs. anaerobic; endurance vs. resistance/strength training) and its duration in patients with compensated and decompensated NASH cirrhosis. Without doubt though, exercise and lifestyle changes need to be tailored to the patient's own ability/competence and confidence, beginning with moderate intensity and maintained for the long term. Simple home-based exercises, using the patient's own body weight for resistance and modified simple aerobic exercises, have recently been shown to be safe and effective in improving physical function and markers of frailty in patients with decompensated cirrhosis. ${ }^{40,41}$ These studies are small, uncontrolled, and not specific to NAFLD, but highlight feasibility in what is a largely neglected field in decompensated cirrhosis.

It must be acknowledged that access to clinical nutritionists or exercise physiotherapists with expertise in the management of patients with chronic liver disease is not universal. There is no structured service provision or reimbursement in place in most health care systems for these services. In areas where they do not exist, it falls on the hepatologist/gastroenterologist to advise patients based on the best available guidelines, ${ }^{34}$ but also to advocate for the services to be provided by health care payers given their real impact on patient outcomes.

\section{Complications of Cirrhosis in Patients with NAFLD}

The development of ascites or encephalopathy in a patient with NASH cirrhosis has major prognostic implications as with other forms of liver disease. Median transplant-free survival in decompensated NASH cirrhosis is $\sim 2$ years. $^{2}$ Physicians should be aware of several distinct challenges in the management of patients with the complications of cirrhosis due to NASH.

\section{Hepatocellular Carcinoma}

The incidence of HCC has increased in the western world over the last two decades. ${ }^{42}$ The increasing prevalence of obesity and T2D have contributed to this rise in patients without NAFLD, ${ }^{43}$ but there is growing evidence of the risk of HCC in NASH cirrhosis. Even though the incidence of HCC in patients with NASH cirrhosis remains lower than in patients with HCV cirrhosis ( 2.6 vs. $4 \%$ in a prospective 4 -year follow-up study), ${ }^{44}$ in countries where the prevalence of viral hepatitis is lower almost $60 \%$ of HCC diagnoses are in patients with a diagnosis of NAFLD. ${ }^{45}$ Indeed, there was a greater than 10 -fold observed increase in the HCC diagnoses in patients with NAFLD in a population-based study in England between 2000 and $2010 .^{42}$ It is therefore not surprising that recent data from the large European Liver Transplant Registry highlighted that HCC was more common in patients transplanted for NASH than in any other disease type. ${ }^{3}$ Once HCC is established in NASH cirrhosis, survival appears to be shorter than their viral hepatitis counterparts. ${ }^{46}$ This may be due to patients with NASH cirrhosis being older, having larger tumors, and being less likely to be diagnosed by surveillance compared with the leaner patients with viral hepatitis. ${ }^{47}$
Ultrasound is the recommended cost-effective modality for HCC screening in patients in with cirrhosis of all etiologies. ${ }^{48,49}$ However, ultrasound has clear limitations for the detection of HCC in patients with elevated BMI and in particular central adiposity. The increased echogenicity of the background liver on ultrasound in patients with NAFLD increases the likelihood in missing smaller lesions. The sensitivity of ultrasound to detect tumors $<3 \mathrm{~cm}$ is substantially reduced in patients with $\mathrm{BMI}>35 \mathrm{~kg} / \mathrm{m}^{2}$ (odds ratio of 0.28 for detection) ${ }^{50}$ In contrast, the sensitivity of magnetic resonance imaging (MRI) to detect curable HCC lesions (i.e., amenable to locoregional treatments, such as resection, radiofrequency ablation, microwave ablation, and cryotherapy) is significantly higher than that of ultrasonography. Despite the limitation of body habitus in a large proportion of patients with NASH, international guidelines still recommend liver ultrasound every 6 months as part of HCC screening by an experienced ultrasonographer. ${ }^{48,49}$ Whether or not (bi-) annual MRI scans (vs. the current ultrasound standard) should be utilized in morbidly obese patients with NASH cirrhosis, who would be suitable for curative locoregional treatments (i.e., Child-Pugh A, good performance status), requires further study and cost-effective analysis.

\section{Portal Vein Thrombosis}

Portal vein thrombosis (PVT) is a common and important complication in patients with cirrhosis. NASH is a prothrombotic condition, as are obesity and diabetes, and the risk of PVT development is higher in NASH cirrhosis (10\%) compared with other causes of cirrhosis (vs. 6\%) in patients listed for liver transplantation in the United States. ${ }^{51}$ The strongest risk factor independently associated with a diagnosis of PVT in over 2,000 patients awaiting liver transplantation was NASH cirrhosis, with an odds ratio of 1.55. Even though the data to support the prophylactic use of low molecular weight heparin (LMWH) in patients with compensated cirrhosis remains controversial, patients with NASH cirrhosis (and no varices) may offer a high-risk group for further clinical studies on anticoagulation and PVT. PVT should be excluded in patients with previously compensated NASH cirrhosis who have a variceal hemorrhage or newly decompensate. A new finding of PVT in this setting will undoubtedly increase the surgical risk of future liver transplantation in cases of NASH cirrhosis, which may already be burdened by heightened cardiovascular and perioperative risk.

Low molecular weight heparin and warfarin are the standard-of-care anticoagulants with the most data to support their use in the treatment of PVT in patients with compensated cirrhosis. Newer direct-acting anticoagulants have not been extensively studied in patients with cirrhosis, but there are numerous case studies that have emerged highlighting their safe use in patients with cirrhosis and PVT. ${ }^{52}$

\section{Ascites and Paracentesis}

Bedside nonultrasound-guided paracentesis or diagnostic taps can be more challenging in patients with NASH cirrhosis and elevated BMI, especially as increased abdominal girth may limit the success of the needle communicating with the peritoneal cavity. At present, there are no data to suggest that 
patients with NASH cirrhosis have more complications after diagnostic or therapeutic paracentesis than other disease types. $^{53}$

\section{Vasoactive Drugs for Variceal Hemorrhage and Hepatorenal Syndrome}

Terlipressin is licensed in Asia, Australasia, and Europe for the management of variceal hemorrhage and hepatorenal syndrome (HRS). It acts as a potent vasoconstrictor to counteract the splanchnic arterial vasodilatation and thereby reduce portal pressures and redirect blood flow (i.e., to the kidneys) in patients with complications of portal hypertension. There are numerous case reports of ischemic complications of terlipressin in the literature, including bowel and peripheral ischemia with skin necrosis. ${ }^{54,55}$ Furthermore, two large trials of bolus terlipressin (doses 8-12 mg per day) in patients with type 1 HRS have reported serious adverse events in 9 to $43 \%$ of patients, with the majority being cardiovascular events. ${ }^{56,57}$ The fact that preexisting coronary artery and peripheral vascular disease increases terlipressin intolerance and adverse events, implies that patients with NASH cirrhosis who have a higher cardiovascular risk profile (vs. other types of cirrhosis) are more susceptible. Interestingly, a recent randomized controlled trial from Italy of 78 patients with type 1 HRS and decompensated cirrhosis highlighted that terlipressin given by continuous infusion is better tolerated and equally efficacious (at lower doses, average $2 \mathrm{mg} /$ day) as intravenous boluses. ${ }^{58}$ Furthermore, in a rather unique outpatient setting in Australia, researchers have shown that continuous infusions of terlipressin for HRS in the community resulted in significant improvements in muscle function and nutritional status, in patients who otherwise would have been expected to decline. ${ }^{59}$ Even though this study was single-center, small $(n=19)$, and had no control arm, it does add to the growing evidence of the benefits of terlipressin by continuous infusion. Together, these studies question whether patients with cirrhosis, HRS, and increased cardiovascular risk, namely those with NASH, should be considered for terlipressin via continuous infusion rather than the current standard of bolus delivery.

\section{Hepatic Encephalopathy}

Patients with NASH cirrhosis are susceptible to hepatic encephalopathy (HE) due to their typically older age than other liver diseases ${ }^{60}$ and slow progressive nature of the disease, thereby enabling revascularization and the development of large portosystemic shunts. Early studies from the 1990s suggested that HE may be more common in patients with NASH cirrhosis (vs. non-NASH) who undergo transjugular intrahepatic portosystemic shunts (TIPS) for variceal bleeding or refractory ascites. ${ }^{61}$ However, this has not been replicated in the past 20 years, with the main predictors of HE post-TIPS being preexisting HE, age, disease severity (i.e., MELD), and most recently sarcopenia. ${ }^{62}$ The presence of HE in patients with NASH cirrhosis often precipitates a vicious cycle of physical frailty (due to poor functional status, poor sleep patterns), unintentional poor compliance with nutritional/dietary advice (due to poor memory), and higher risk of recurrent admissions to hospital. It is not surprising that previous studies have reported that malnutrition, low sodium, and cryptogenic cirrhosis (which may be "burnt out" NASH) are associated with increased mortality on the transplant waiting list. ${ }^{63}$ Actively screening for minimal HE and prompt management (lactulose, rifaximin, nutrition) in patients with NASH cirrhosis is critical in maintaining their performance status and preventing further frailty.

\section{Extrahepatic Comorbidities}

Metabolic comorbidities are common in patients with NAFLD and tend to correlate with disease severity. The gamut of these manifestations that drive insulin resistance and NAFLD disease severity include obesity and visceral adiposity, T2D mellitus (T2DM), sleep apnea, polycystic ovarian syndrome, and other endocrine disorders (such as hypogonadism, hypopituitarism). ${ }^{64-66}$

\section{Type 2 Diabetes}

Type 2 diabetes mellitus is common in patients with cirrhosis irrespective of etiology and is an independent risk factor for HCC development. ${ }^{67,68}$ All patients with NASH cirrhosis should be screened for T2DM by fasting or random blood glucose or the oral glucose tolerance test. ${ }^{69}$ Glycated hemoglobin A1c (HBA1c) is a reliable test to assess chronic glycemia in patients with T2DM, but has suboptimal performance in patients with cirrhosis. ${ }^{70}$ A normal HbA1c may not necessarily rule out T2DM in the setting of cirrhosis and in patients with decompensated cirrhosis. ${ }^{70}$ Fructosamine may be a better marker to assess glycemic control in patients with cirrhosis and T2DM, ${ }^{71}$ but validated laboratories cut-offs do not exist at present. With the exception of metformin, most of the established, older antiglycemic medications are weight-promoting (i.e., glicazides, thiazolidinediones, insulin), which may in turn worsen adiposity and potentially portal hypertension in NASH cirrhosis. GLP-1 agonists, dipeptidyl peptidase- 4 inhibitors, and sodium-glucose cotransporter-2 inhibitors represent promising groups of agents to manage T2DM in NASH as they promote weight loss or are weight-neutral, respectively. There remains a lack of long-term safety and efficacy data of these drugs in patients with cirrhosis though, with future randomized controlled trials ongoing. However, for patients with poorly controlled T2DM and decompensated cirrhosis secondary to NASH, insulin remains the mainstay of therapy.

\section{Renal Disease}

The presence and severity of NASH are associated with an increased risk and severity of chronic kidney disease (CKD), with advanced fibrosis/cirrhosis associated with higher prevalence (odds ratio, 5). ${ }^{72}$ This is largely due to the coexistence of hypertension and T2DM, even though a 53study sized meta-analysis highlighted that the magnitude of the risk of CKD with NASH-advanced fibrosis/cirrhosis was maintained after controlling for all other renal risk factors. This emphasizes the importance of the need for lifestyle interventions, the pursuit of smoking cessation, the use of statins, and possible angiotensin receptor blockers (ARBs) (especially in the presence of proteinuria) in patients with 
NASH cirrhosis. It is important to highlight, however, that the use of statins and ARBs in patients who progress to decompensated disease may cause more harm (vs. benefit) and should be stopped immediately.

\section{Cardiovascular Disease}

Patients with NASH cirrhosis are at increased risk of cardiovascular disease and cardiac events. A recent meta-analysis suggested that NAFLD was associated with a $65 \%$ increased risk of developing fatal and nonfatal cardiac events over a median follow-up period of 7 years, and this risk was higher with increased severity of liver disease. ${ }^{73}$ There are conflicting data on whether NASH is an independent risk factor for cardiovascular disease, or if the increased risk is related to the metabolic comorbidities in these patients alone. The spectrum of cardiovascular disease associated with NAFLD includes coronary artery disease, left ventricular dysfunction/hypertrophy, diastolic dysfunction, heart failure, atrial fibrillation, and QTc prolongation. ${ }^{74}$ The implications of such in the transplant work-up are discussed below.

\section{Extrahepatic Malignancy}

Patients with NAFLD are at increased risk for extrahepatic malignancies. Uterine (relative risk [RR], 2.39), gastric (RR, $2.34)$, pancreatic ( $R R, 2.09$ ), and colon cancer ( $R R, 1.76)$ are more common in patients with NAFLD compared with an age- and sex-matched population over a median follow-up of 8 years. ${ }^{75}$ Due to the majority of the evidence being collated from retrospective cohort studies, a causative effect of NASH cirrhosis and extrahepatic malignancy cannot be determined. This results in heterogeneity in management; an example of such is the high nonselective uptake and absence of colonoscopy screening in the transplant assessment in the United States and Europe, respectively. The combination of NASH cirrhosis (plus accompanying metabolic risks) and a new diagnosis of intra-abdominal malignancy often results in limited therapeutic options. Calculators, such as the Mayo postoperative mortality risk prediction model, have been developed to aid clinicians with the risk assessment of cancer surgery. However, in general only those patients with compensated Child-Pugh A cirrhosis, absent portal hypertension, and controlled metabolic risk factors are likely to survive general anesthesia and subsequent surgery.

\section{Liver Transplantation for NASH}

NASH cirrhosis is now the second most common indication for liver transplant listing in the United States, and the proportion of liver transplants for NASH cirrhosis is only likely to further increase. ${ }^{2}$

\section{Liver Transplant Assessment}

NASH patients referred for transplantation tend to be older than patients with HCV cirrhosis with more comorbidities despite similar MELD scores. ${ }^{11,76}$ Analysis from the United Network for Organ Sharing (UNOS) database suggests that NASH patients are not disadvantaged by higher waitlist mortality or lower transplantation rates once listed for liver transplantation. However, there is a reported bias toward patients with NASH cirrhosis when it comes to listing for transplantation. Patients with NASH cirrhosis are nearly three times more likely to be turned down for transplant listing due to comorbid conditions than patients with viral hepatitis. ${ }^{77}$ Another single-center study reported that patients with NASH cirrhosis were more likely to be declined for transplant even when controlling for comorbidities. Patients with NASH cirrhosis declined for liver transplantation are more likely to die from their liver disease and not their comorbid conditions. ${ }^{11}$

Cardiovascular disease remains a leading cause of posttransplant death in patients with NASH cirrhosis. The decision making regarding transplant listing in patients with $\mathrm{NASH}$ cirrhosis is complicated by the lack of consensus regarding the optimal pretransplant cardiovascular assessment for patients with NASH cirrhosis. Consensus guidelines do not recommend a specific cardiovascular disease risk evaluation for NASH patients which is generally determined by local expertise. $^{78}$ It is standard practice that all patients being assessed for transplant undergo noninvasive assessment with electrocardiography and echocardiography. If these are abnormal, further investigations are recommended and a cardiology review. These further investigations are patient- and centerspecific, and may include cardiopulmonary exercise testing, stress echocardiogram, myocardial perfusion imaging by single photon emission tomography, or cardiac computed tomography. Invasive investigations such as coronary angiography may be warranted on a case-by-case basis in patients with abnormal noninvasive assessments. Several centers perform coronary angiography as a primary investigation in transplant assessment, ${ }^{79}$ but studies using this strategy are generally retrospective in nature and may be subject to selection bias. The aim of cardiac assessment is to diagnose patients with severe coronary artery disease that clearly precludes transplantation. Patients with established coronary artery disease who require revascularization with coronary stenting or bypass should be discussed on a case-by-case basis with the involvement of cardiologists and anesthetics with expertise in the high-risk patients.

Significant obesity has previously been considered as a contraindication to transplantation, with studies from the pre-MELD suggesting poorer transplant outcomes in this group of patients. ${ }^{80} \mathrm{BMI}>40 \mathrm{~kg} / \mathrm{m}^{2}$ alone is not a contraindication, but the UNOS data demonstrate noninferior posttransplant outcomes in highly selected patients with BMI $>40 \mathrm{~kg} / \mathrm{m}^{2}$ who undergo transplant, but this group did have higher mortality on the waiting list. ${ }^{81}$ Therefore, patients with BMI $>40 \mathrm{~kg} / \mathrm{m}^{2}$ with comorbidities, particularly concurrent diabetes, should undergo careful assessment by a multidisciplinary transplant team with expertise in transplanting patients with morbid obesity. ${ }^{79}$ The optimal timing for bariatric surgery (at the time of transplant or deferred) is still unknown, but should not be considered in patients with NASH cirrhosis who have clinically significant portal hypertension prior to transplantation.

\section{Postliver Transplant care}

Patients transplanted for NASH cirrhosis have similar posttransplant survival compared with other etiologies of cirrhosis undergoing transplant. ${ }^{79}$ Data from the Scientific Registry of 
Transplant Recipients estimated 1- and 3-year posttransplant survival for NASH cirrhosis as 84 and $78 \%$, respectively. ${ }^{82}$ Cardiovascular disease contributes to a higher proportion of posttransplant deaths in patients transplanted for NASH cirrhosis compared with non-NASH patients who undergo transplant. ${ }^{83}$ However, patients with NASH cirrhosis are at a lower risk of graft failure following liver transplantation.

Immunosuppression with calcineurin inhibitors and steroids may result in worsening of glycemic control in the posttransplant period in NASH patients with or without concomitant diabetes. These agents also promote weight gain which may increase the risk of recurrent NASH in the liver allograft. It may be prudent to rationalize and individualize immunosuppression in patients with NASH cirrhosis to mitigate these effects, while being vigilant to the risk of acute cellular rejection in the posttransplant period. The optimal immunosuppressive strategy in these patients is yet to be defined, and requires further prospective research studies.

Proactive management of cardiovascular risk factors is important in the posttransplant setting (good glycemic, blood pressure control, and statin therapy for hyperlipidemia) to reduce the risk of cardiovascular events posttransplant.

\section{Conclusion}

Patients with NASH cirrhosis require a multidisciplinary approach to management with close involvement of clinical nutritionists, exercise physiotherapists, metabolic/endocrine specialists, and hepatologists/gastroenterologists. This is best achieved in specialist settings that address not only the management of liver disease, but their wider metabolic risk factors given the higher risk of cardiovascular disease and malignancy in these groups of patients. It is no longer best practice to simply monitor for the progression of liver disease alone. Patients with NASH cirrhosis should be considered for early referral for liver transplant assessment especially if they exhibit early signs of clinically significant portal hypertension (varices or ascites) or decompensation, as their outcomes post liver transplant are on par with other forms of liver disease if appropriately selected for transplant.

\section{Authors' Contributions}

O.E.S. and M.J.A. performed the literature search. O.E.S. wrote the first draft. M.J.A. edited the draft and approved the final manuscript.

Conflicts of Interest

M.J.A. has received research grants from Novo Nordisk Ltd, Mumbai.

\section{References}

1 Kabbany MN, Conjeevaram Selvakumar PK, Watt K, et al. Prevalence of nonalcoholic steatohepatitis-associated cirrhosis in the United States: an analysis of National Health and Nutrition Examination Survey data. Am J Gastroenterol 2017;112(04):581-587

2 Agopian VG, Kaldas FM, Hong JC, et al. Liver transplantation for nonalcoholic steatohepatitis: the new epidemic. Ann Surg 2012; 256(04):624-633
3 Haldar D, Kern B, Hodson J, et al; European Liver and Intestine Transplant Association (ELITA). Outcomes of liver transplantation for non-alcoholic steatohepatitis: a European Liver Transplant Registry study. J Hepatol 2019;71(02):313-322

4 Ludwig J, Viggiano TR, McGill DB, Oh BJ. Nonalcoholic steatohepatitis: Mayo Clinic experiences with a hitherto unnamed disease. Mayo Clin Proc 1980;55(07):434-438

5 Singh S, Allen AM, Wang Z, Prokop LJ, Murad MH, Loomba R. Fibrosis progression in nonalcoholic fatty liver vs nonalcoholic steatohepatitis: a systematic review and meta-analysis of pairedbiopsy studies. Clin Gastroenterol Hepatol 2015;13(04):643-54. e1, 9, quiz e39-e40

6 Hagström H, Nasr P, Ekstedt M, et al. Fibrosis stage but not NASH predicts mortality and time to development of severe liver disease in biopsy-proven NAFLD. JHepatol 2017;67(06):1265-1273

7 Bosch J, Harrison SA, Abdelmalek MF, et al. Hepatic venous pressure gradient (HVPG) predicts clinic disease progression in patients with compensated cirrhosis due to NASH. Hepatology 2017;66:244A

8 Bertolotti M, Lonardo A, Mussi C, et al. Nonalcoholic fatty liver disease and aging: epidemiology to management. World J Gastroenterol 2014;20(39):14185-14204

9 Sanyal AJ, Banas C, Sargeant C, et al. Similarities and differences in outcomes of cirrhosis due to nonalcoholic steatohepatitis and hepatitis C. Hepatology 2006;43(04):682-689

10 Haflidadottir S, Jonasson JG, Norland H, et al. Long-term follow-up and liver-related death rate in patients with non-alcoholic and alcoholic related fatty liver disease. BMC Gastroenterol 2014; $14: 166$

11 O’Leary JG, Landaverde C, Jennings L, Goldstein RM, Davis GL. Patients with NASH and cryptogenic cirrhosis are less likely than those with hepatitis $C$ to receive liver transplants. Clin Gastroenterol Hepatol 2011;9(08):700-704.e1

12 Moriya A, Iwasaki Y, Ohguchi S, et al. Alcohol consumption appears to protect against non-alcoholic fatty liver disease. Aliment Pharmacol Ther 2011;33(03):378-388

13 Ekstedt M, Franzén LE, Holmqvist M, et al. Alcohol consumption is associated with progression of hepatic fibrosis in non-alcoholic fatty liver disease. Scand J Gastroenterol 2009;44(03):366-374

14 Ratziu V. A critical review of endpoints for non-cirrhotic NASH therapeutic trials. J Hepatol 2018;68(02):353-361

15 Gilead Sciences Press Release. Available at: https://www.gilead.com/news-and-press/press-room/press-releases/2019/2/gileadannounces-topline-data-from-phase-3-stellar4-study-of-selonsertib-in-compensated-cirrhosis-f4-due-to-nonalcoholic-steatohepatitis-nash. Accessed February 11, 2019

16 Garcia-Tsao G, Bosch J, Kayali Z , et al. Double-blind, placebocontrolled, randomised trial of emricasan in subjects with NASH cirrhosis and severe portal hypertension. EASL International Liver Congress, Vienna, April 2019

17 Afdhal N, Everson GT, Calleja JL, et al. Effect of viral suppression on hepatic venous pressure gradient in hepatitis $\mathrm{C}$ with cirrhosis and portal hypertension. JViral Hepat 2017;24(10):823-831

18 Harrison SA, Abdelmalek MF, Caldwell S, et al; GS-US-321-0105 and GS-US-321-0106 Investigators. Simtuzumab is ineffective for patients with bridging fibrosis or compensated cirrhosis caused by non-alcoholic steatohepatitis. Gastroenterology 2018;155 (04):1140-1153

19 Rockey DC. Noninvasive assessment of liver fibrosis and portal hypertension with transient elastography. Gastroenterology 2008;134(01):8-14

20 Castéra L, Foucher J, Bernard PH, et al. Pitfalls of liver stiffness measurement: a 5-year prospective study of 13,369 examinations. Hepatology 2010;51(03):828-835

21 Wong VW, Vergniol J, Wong GL, et al. Liver stiffness measurement using XL probe in patients with nonalcoholic fatty liver disease. Am J Gastroenterol 2012;107(12):1862-1871 
22 Singh S, Muir AJ, Dieterich DT, Falck-Ytter YT. American Gastroenterological Association Institute technical review on the role of elastography in chronic liver diseases. Gastroenterology 2017; 152(06):1544-1577

23 Xiao G, Zhu S, Xiao X, Yan L, Yang J, Wu G. Comparison of laboratory tests, ultrasound, or magnetic resonance elastography to detect fibrosis in patients with nonalcoholic fatty liver disease: a meta-analysis. Hepatology 2017;66(05):1486-1501

24 Castera L, Friedrich-Rust M, Loomba R. Non-invasive assessment of liver disease in patients with non-alcoholic fatty liver disease. Gastroenterology 2019;156(05):1264-1281.e4

25 de Franchis R, Baveno VI; Baveno VI Faculty. Expanding consensus in portal hypertension: report of the Baveno VI Consensus Workshop: stratifying risk and individualizing care for portal hypertension. J Hepatol 2015;63(03):743-752

26 Petta S, Sebastiani G, Bugianesi E, et al. Non-invasive prediction of esophageal varices by stiffness and platelet in non-alcoholic fatty liver disease cirrhosis. J Hepatol 2018;69(04):878-885

27 Shearer J, Chizhande D, Jones R, Rowe IA. Non-invasive prediction of oesophageal varices in patients with cirrhosis secondary to non-alcoholic fatty liver disease. JHepatol 2018;69(05): 1202-1203

28 Cabassa P, Ravanelli M, Rossini A, Contessi G, Almajdalawi R, Maroldi R. Acoustic radiation force impulse quantification of spleen elasticity for assessing liver fibrosis. Abdom Imaging 2015;40(04):738-744

29 Wong GL, Liang LY, Kwok R, et al. Low risk of variceal bleeding in patients with cirrhosis after variceal screening stratified by liver/spleen stiffness. Hepatology 2019. Doi: 10.1002/hep.30522

30 Lai JC, Feng S, Terrault NA, Lizaola B, Hayssen H, Covinsky K. Frailty predicts waitlist mortality in liver transplant candidates. Am J Transplant 2014;14(08):1870-1879

31 Lai JC, Dodge JL, Sen S, Covinsky K, Feng S. Functional decline in patients with cirrhosis awaiting liver transplantation: results from the functional assessment in liver transplantation (FrAILT) study. Hepatology 2016;63(02):574-580

32 Merli M, Lattanzi B, Aprile F. Sarcopenic obesity in fatty liver. Curr Opin Clin Nutr Metab Care 2019;22(03):185-190

33 Berzigotti A, Garcia-Tsao G, Bosch J, et al; Portal Hypertension Collaborative Group. Obesity is an independent risk factor for clinical decompensation in patients with cirrhosis. Hepatology 2011;54(02):555-561

34 European Association for the Study of the Liver. Electronic address: easloffice@easloffice.eu; European Association for the Study of the Liver. EASL Clinical Practice Guidelines on nutrition in chronic liver disease. J Hepatol 2019;70(01):172-193

35 Saab S, Lalezari D, Pruthi P, Alper T, Tong MJ. The impact of obesity on patient survival in liver transplant recipients: a meta-analysis. Liver Int 2015;35(01):164-170

36 Hayashi F, Momoki C, Yuikawa M, et al. Nutritional status in relation to lifestyle in patients with compensated viral cirrhosis. World J Gastroenterol 2012;18(40):5759-5770

37 Vilar-Gomez E, Martinez-Perez Y, Calzadilla-Bertot L, et al. Weight loss through lifestyle modification significantly reduces features of nonalcoholic steatohepatitis. Gastroenterology 2015;149(02): 367-78.e5, quiz e14-e15

38 Pi-Sunyer X, Blackburn G, Brancati FL, et al; Look AHEAD Research Group. Reduction in weight and cardiovascular disease risk factors in individuals with type 2 diabetes: one-year results of the look AHEAD trial. Diabetes Care 2007;30(06):1374-1383

39 Berzigotti A, Albillos A, Villanueva C, et al; Ciberehd SportDiet Collaborative Group. Effects of an intensive lifestyle intervention program on portal hypertension in patients with cirrhosis and obesity: the SportDiet study. Hepatology 2017;65(04):1293-1305

40 Kruger C, McNeely ML, Bailey RJ, et al. Home exercise training improves exercise capacity in cirrhosis patients: role of exercise adherence. Sci Rep 2018;8(01):99
41 Williams FR, Vallance A, Faulkner T, et al. Home-based exercise in patients awaiting liver transplantation: a feasibility study. Liver Transpl 2019;25(07):995-1006

42 Dyson J, Jaques B, Chattopadyhay D, et al. Hepatocellular cancer: the impact of obesity, type 2 diabetes and a multidisciplinary team. JHepatol 2014;60(01):110-117

43 El-Serag HB, Tran T, Everhart JE. Diabetes increases the risk of chronic liver disease and hepatocellular carcinoma. Gastroenterology 2004;126(02):460-468

44 Ascha MS, Hanouneh IA, Lopez R, Tamimi TA, Feldstein AF, Zein $\mathrm{NN}$. The incidence and risk factors of hepatocellular carcinoma in patients with nonalcoholic steatohepatitis. Hepatology 2010;51 (06):1972-1978

45 Sanyal A, Poklepovic A, Moyneur E, Barghout V. Population-based risk factors and resource utilization for HCC: US perspective. Curr Med Res Opin 2010;26(09):2183-2191

46 Guzman G, Brunt EM, Petrovic LM, Chejfec G, Layden TJ, Cotler SJ. Does nonalcoholic fatty liver disease predispose patients to hepatocellular carcinoma in the absence of cirrhosis? Arch Pathol Lab Med 2008;132(11):1761-1766

47 Calzadilla Bertot L, Adams LA. The natural course of non-alcoholic fatty liver disease. Int J Mol Sci 2016;17(05):774

48 European Association for the Study of the Liver. Electronic address: easloffice@easloffice.eu; European Association for the Study of the Liver. Management of hepatocellular carcinoma. J Hepatol 2018;69(01):182-236

49 Heimbach JK, Kulik LM, Finn RS, et al. AASLD guidelines for the treatment of hepatocellular carcinoma. Hepatology 2018;67(01): 358-380

50 Wong LL, Reyes RJ, Kwee SA, Hernandez BY, Kalathil SC, Tsai NC. Pitfalls in surveillance for hepatocellular carcinoma: how successful is it in the real world? Clin Mol Hepatol 2017;23(03): 239-248

51 Stine JG, Shah NL, Argo CK, Pelletier SJ, Caldwell SH, Northup PG. Increased risk of portal vein thrombosis in patients with cirrhosis due to nonalcoholic steatohepatitis. Liver Transpl 2015;21(08): 1016-1021

52 Weinberg EM, Palecki J, Reddy KR. Direct-acting oral anticoagulants (DOACs) in cirrhosis and cirrhosis-associated portal vein thrombosis. Semin Liver Dis 2019;39(02):195-208

53 De Gottardi A, Thévenot T, Spahr L, et al. Risk of complications after abdominal paracentesis in cirrhotic patients: a prospective study. Clin Gastroenterol Hepatol 2009;7(08):906-909

54 Sarma P, Muktesh G, Dhaka N, et al. Terlipressin-induced peripheral ischaemic gangrene in a diabetic patient. JPharmacol Pharmacother 2017;8(03):148-150

55 Elzouki AN, El-Menyar A, Ahmed E, Elbadri ME, Imam YZ, Gurbanna BA. Terlipressin-induced severe left and right ventricular dysfunction in patient presented with upper gastrointestinal bleeding: case report and literature review. Am J Emerg Med 2010;28(04):540.e1-540.e6

56 Sanyal AJ, Boyer T, Garcia-Tsao G, et al; Terlipressin Study Group. A randomized, prospective, double-blind, placebo-controlled trial of terlipressin for type 1 hepatorenal syndrome. Gastroenterology 2008;134(05):1360-1368

57 Martín-Llahí M, Pépin MN, Guevara M, et al; TAHRS Investigators. Terlipressin and albumin vs albumin in patients with cirrhosis and hepatorenal syndrome: a randomized study. Gastroenterology 2008;134(05):1352-1359

58 Cavallin M, Piano S, Romano A, et al. Terlipressin given by continuous intravenous infusion versus intravenous boluses in the treatment of hepatorenal syndrome: a randomized controlled study. Hepatology 2016;63(03):983-992

59 Chapman B, Gow P, Sinclair M, et al. Continuous terlipressin infusion is associated with improved diet intake and muscle strength in patients awaiting liver transplant. JHep Reports 2019;1(02):107-113 
60 Dasani BM, Sigal SH, Lieber CS. Analysis of risk factors for chronic hepatic encephalopathy: the role of Helicobacter pylori infection. Am J Gastroenterol 1998;93(05):726-731

61 Somberg KA, Riegler JL, LaBerge JM, et al. Hepatic encephalopathy after transjugular intrahepatic portosystemic shunts: incidence and risk factors. Am J Gastroenterol 1995;90(04):549-555

62 Dhaliwal A, Armstrong MJ, Tripathi D. Patient selection for transjugular intrahepatic portosystemic shunt (TIPSS) insertion in variceal bleeding and refractory ascites. Curr Hepatol Rep 2017; 16:241

63 Ferreira LG, Anastácio LR, Lima AS, Touslon Davisson Correia MI. Predictors of mortality in patients on the waiting list for liver transplantation. Nutr Hosp 2013;28(03):914-919

64 Bedogni G, Miglioli L, Masutti F, Tiribelli C, Marchesini G, Bellentani S. Prevalence of and risk factors for nonalcoholic fatty liver disease: the Dionysos nutrition and liver study. Hepatology 2005; 42(01):44-52

65 Caldwell SH, Crespo DM. The spectrum expanded: cryptogenic cirrhosis and the natural history of non-alcoholic fatty liver disease. J Hepatol 2004;40(04):578-584

66 Aron-Wisnewsky J, Minville C, Tordjman J, et al. Chronic intermittent hypoxia is a major trigger for non-alcoholic fatty liver disease in morbid obese. J Hepatol 2012;56(01):225-233

67 Vernon G, Baranova A, Younossi ZM. Systematic review: the epidemiology and natural history of non-alcoholic fatty liver disease and non-alcoholic steatohepatitis in adults. Aliment Pharmacol Ther 2011;34(03):274-285

68 Loomba R, Abraham M, Unalp A, et al; Nonalcoholic Steatohepatitis Clinical Research Network. Association between diabetes, family history of diabetes, and risk of nonalcoholic steatohepatitis and fibrosis. Hepatology 2012;56(03):943-951

69 European Association for the Study of the Liver (EASL). European Association for the Study of Diabetes (EASD); European Association for the Study of Obesity (EASO). EASL-EASD-EASO Clinical Practice Guidelines for the management of non-alcoholic fatty liver disease. JHepatol 2016;64:1388-1402

70 Addepally NS, George N, Martinez-Macias R, Garcia-Saenz-deSicilia M, Kim WR, Duarte-Rojo A. Hemoglobin A1c has suboptimal performance to diagnose and monitor diabetes mellitus in patients with cirrhosis. Dig Dis Sci 2018;63(12): 3498-3508

71 Lahousen T, Hegenbarth K, Ille R, et al. Determination of glycated hemoglobin in patients with advanced liver disease. World J Gastroenterol 2004;10(15):2284-2286

72 Musso G, Tabibian JH, Charlton M. Chronic kidney disease (CKD) and NAFLD: time for awareness and screening. JHepatol 2015;62 (04):983-984
73 Targher G, Byrne CD, Lonardo A, Zoppini G, Barbui C. Nonalcoholic fatty liver disease and risk of incident cardiovascular disease: a meta-analysis. J Hepatol 2016;65(03):589-600

74 Lonardo A, Nascimbeni F, Mantovani A, Targher G. Hypertension, diabetes, atherosclerosis and NASH: cause or consequence? J Hepatol 2018;68(02):335-352

75 Hicks S, Mara K, Larson JL, et al. The incidence of extrahepatic malignancies in non-alcoholic fatty liver disease (NAFLD). AASLD Liver Meeting 2018, San Francisco, Abstract 0031

76 Ahn JS, Sinn DH, Min YW, et al. Non-alcoholic fatty liver diseases and risk of colorectal neoplasia. Aliment Pharmacol Ther 2017;45 (02):345-353

77 Danford CJ, Iriana S, Shen C, Curry MP, Lai M. Evidence of bias during liver transplant evaluation of non-alcoholic steatohepatitis cirrhosis patients. Liver Int 2019;39(06):1165-1173

78 Thuluvath PJ, Hanish S, Savva Y. Waiting list mortality and transplant rates for NASH cirrhosis when compared with cryptogenic alcoholic, or AIH cirrhosis. Transplantation 2019;103(01):113-121

79 Tsochatzis E, Coilly A, Nadalin S, et al. International Liver Transplantation consensus statement on end-stage liver disease due to nonalcoholic steatohepatitis and liver transplantation. Transplantation 2019;103(01):45-56

80 Maddur H, Bourdillon PD, Liangpunsakul S, et al. Role of cardiac catheterization and percutaneous coronary intervention in the preoperative assessment and management of patients before orthotopic liver transplantation. Liver Transpl 2014;20(06):664-672

81 Nair S, Verma S, Thuluvath PJ. Obesity and its effect on survival in patients undergoing orthotopic liver transplantation in the United States. Hepatology 2002;35(01):105-109

82 Schlansky B, Naugler WE, Orloff SL, Enestvedt CK. Higher mortality and survival benefit in obese patients awaiting transplantation. Transplantation 2016;100(12):2648-2655

83 Charlton MR, Burns JM, Pedersen RA, Watt KD, Heimbach JK, Dierkhising RA. Frequency and outcomes of liver transplantation for nonalcoholic steatohepatitis in the United States. Gastroenterology 2011;141(04):1249-1253

84 Angulo P, Kleiner DE, Dam-Larsen S, et al. Liver fibrosis, but no other histologic features, is associated with long-term outcomes of patients with non-alcoholic fatty liver disease. Gastroenterology 2015;149(02):389-97.e10

85 Younossi ZM, Stepanova M, Rafiq N, et al. Pathologic criteria for nonalcoholic steatohepatitis: interprotocol agreement and ability to predict liver-related mortality. Hepatology 2011;53(06):1874-1882

86 Sebastiani G, Alshaalan R, Wong P, et al. Prognostic value of noninvasive fibrosis and steatosis tools, hepatic venous pressure gradient (HVPG) and histology in non-alcoholic steatohepatitis. PLoS One 2015;10(06):e0128774 